Revue d'histoire de l'Amérique française

ZWB REVUE D.HISTOIRE DE L'AMÉRIQUE FRANÇAISE

\title{
Prise de contact de La Galissonière avec la Nouvelle-France
}

\section{Roland Lamontagne}

Volume 14, numéro 3, décembre 1960

URI : https://id.erudit.org/iderudit/302062ar

DOI : https://doi.org/10.7202/302062ar

Aller au sommaire du numéro

Éditeur(s)

Institut d'histoire de l'Amérique française

ISSN

0035-2357 (imprimé)

1492-1383 (numérique)

Découvrir la revue

Citer cet article

Lamontagne, R. (1960). Prise de contact de La Galissonière avec la

Nouvelle-France. Revue d'histoire de l'Amérique française, 14(3), 384-394.

https://doi.org/10.7202/302062ar d'utilisation que vous pouvez consulter en ligne.

https://apropos.erudit.org/fr/usagers/politique-dutilisation/ 


\section{PRISE DE CONTACT DE LA GALISSONIËRE AVEC LA NOUVELLE-FRANCE}

Le ministre de la Marine Jean-Frédéric Phélypeaux, comte de Maurepas, annonce à Roland-Michel Barrin de La Galissonière, le 6 juin 1747, qu'il se présente une « occasion fort intéressante 》 de donner de nouvelles preuves d'attachement au service du roi. Il n'a pas hésité, affirme-t-il, à assurer Louis XV du dévouement de La Galissonière. Il relate les «fâcheuses nouvelles » relatives à l'escadre de Pierre-Jacques Taffanel, marquis de La Jonquière. ${ }^{1}$ La flotte de l'amiral George Anson était composée de quatorze vaisseaux de ligne et d'une frégate. La Jonquière ne disposait que de six vaisseaux et d'une frégate. Il apportait des secours à la colonie et il devait succéder à Charles de La Boische, marquis de Beauharnais, à titre de gouverneur général. La Jonquière fut blessé et amené en captivité en Angleterre. ${ }^{2}$

Afin de pallier les effets de cette «malheureuse aventure », le roi décide d'envoyer de nouveaux secours au Canada. Ses vaisseaux le Northumberland et l'Alcyon ainsi que la flûte la Gironde transporteront des armes et des munitions de guerre, des marchandises de Rochefort, de Bordeaux et de La Rochelle, des vins et des alcools provenant des magasins du munitionnaire et une nouvelle provision de sel des fournisseurs ordinaires. «Mais le secours le plus important et dont la Colonie se trouve avoir le plus besoin c'est un chef en etat de la conduire, et de la deffendre. 》

1 «Les Anglois embarqués sur le $\mathrm{N}^{\text {re }}$ le Grand Scypion de la Rochelle repris par un Corsaire de $\mathbf{S}^{t}$ Malo et conduit a Morlaix, annoncent la prise du Serieux, de la Gloire et de l'Emeraude. Un autre Navire de notre flotte relaché a la Corogne ne parle que d'une fregate de 40. Canons. Mais tout s'accorde sur la grande Superiorité de l'Escad. Angloise; de maniere qu'il faut s'attendre que quelque chose qu'ait pu faire la nôtre, elle aura eté accablée par le nombre. 》 Maurepas à La Galissonière, Paris, 6 juin 1747, AC, B86: 335.

2 Guy Frégault, François Bigot, administrateur français (2 vol., Montréal, 1948), 1: 302; Basil Williams, The Whig Supremacy, 1714-1760 (Oxford, 1952), 248. 
La Galissonière avait déjà fait représenter au ministre Maurepas que son inclination le portait plutôt à commander des vaisseaux qu'à être gouverneur, et «qu'il désiroit ne faire son chemin dans la Marine que par cette voie ...». ${ }^{3}$ Il venait à peine d'être nommé commandant du Monarque que sa destination était changée. Le roi avait jugé à propos de lui confier le commandement de la Nouvelle-France pendant la captivité de La Jonquière. La Galissonière reste libre de refuser. Le 14 juin 1747, Maurepas précise l'état provisoire de cette mission. ${ }^{4}$ Outre les lettres patentes, ${ }^{5}$ il adresse à Roland-Michel Barrin de La Galissonière une copie des instructions qu'avait reçues La Jonquière pour le mettre en état de remplir les divers objets de ce commandement. ${ }^{6}$

La Galissonière était venu en Nouvelle-France, à maintes reprises, comme il le note dans la lettre qu'il adressait à Mère Sainte-Hélène [Marie-Andrée Regnard Duplessis], supérieure de l'Hôtel-Dieu de Québec, le 7 septembre 1747. " Je suis venu ici assés souvent, Madame, pour Conoitre votre communauté et pour prendre pour elle les sentimens d'estime qu'elle merite. $\gg^{7}$ Précédé d'une journée par l'Alcyon et la Gironde, le Northumberland atteignait Québec, le 19 septembre 1747. Le gouverneur intérimaire débarquait vers quatre heures de l'après-midi. ${ }^{8} \mathrm{Il}$ fut l'objet des cérémonies qui marquaient l'arrivée des gouver-

3 Jean-Paul Grandjean de Fouchy, «Eloge de M. le Marquis de La Galissonière 》, Histoire de l'Académie Royale des Sciences, année 1756, 150.

4 \& M. de la Jonquiere étant pourvû du Gouvernement general, il n'est pas possible pour le present de vous en expedier les provisions; mais les lettres de commandement vous donnent les mêmes pouvoirs, les mêmes droits, la même autorité et les mêmes honneurs attachés a la charge de Geur $\mathrm{L}^{\mathrm{t}}$ general; et vous joüirés des mêmes appointements. 》 Maurepas à La Galissonière, Pontchartrain, 14 juin 1747, B 86: 356-357.

5 Lettres Patentes pour donner le Commandement Général de la Nouvelle-France, au sieur Comte de La Galissonière, du $10^{\mathrm{e}}$ juin 1747, Edits et Ordonnances, 3: 73-75. 349.

6 Maurepas à La Galissonière, Pontchartrain, 13 juin 1747, AC, B 86 :

7 La Galissonière, a bord du Northumberland, le 7 septembre 1747. Dépôt d'archives des Augustines de l'Hôtel-Dieu de Québec.

8 « Extrait en forme de journal, de ce qui s'est passé d'intéressant dans la colonie à l'occasion des mouvements de guerre et des différents avis reçus depuis le départ des vaisseaux au mois de novembre $1746 », \mathrm{AC}, \mathrm{C} 11 \mathrm{~A}$, 87-1: 232 . 
neurs généraux. En même temps qu'il communiquait au ministre le compte rendu de la réception, l'intendant Gilles Hocquart témoignait de bons sentiments à l'égard du représentant du roi. ${ }^{9}$

Roland-Michel Barrin de La Galissonière prend connaissance des problèmes de la colonie. Ses premières dépêches au ministre portent la signature conjointe de l'intendant. Elles contiennent de nombreux détails dont nous retiendrons ceux qui mettent en relief la contribution du gouverneur.

Faute de ressources, le gouverneur général Beauharnais avait rappelé un détachement de trois cents hommes qui avaient passé un an, en Acadie.

Les 300 hommes qui avoient resté à l'Acadie et qui y ont passé l'hyver en sont revenus l'esté dernier, que Monsieur le Marquis de Beauharnois les rappela, il n'estoit plus possible de les y laisser plus longtemps par le manque de vivres, d'argent et de marchandises; d'ailleurs ce détachement extrêmement fatigué d'une campagne d'un an, où ils ont esté continuellement employés de poste en poste avoit besoin d'estre relevé, sans les autres considérations qui ont déterminé à prendre ce party, telles sont la crainte d'estre enveloppez par des ennemis nombreux et l'inutilité dont ils seroient, Sa Majesté paroissant avoir abandonné, du moins pour quelques temps son projet sur l'Accadie. ${ }^{10}$

Peu après son arrivée à Québec, La Galissonière discutait avec Gilles Hocquart de la préparation d'une «nouvelle entreprise » en Acadie. Ce projet ne fut pas exécuté; la saison était trop avancée. Le gouverneur explique ses intentions aux missionnaires Maillard et Germain sur la conduite que les sauvages de Cobequid et de Beaubassin doivent tenir «pour faire des

9 « Je vous crois bien persuadé, Monseigneur, de mon attention a concourir avec M. de La Galissonniere et a le seconder dans toutes les affaires de l'administration générale, j'ay l'honneur de le connoistre depuis longtemps, rien ne troublera, a ce que j'espere, la bonne intelligence qui doit regner parmi nous; nous avons commencé a travailler ensemble et a nous communiquer réciproquement nos vües pour le bien et l'avantage du service du Roy et de la colonie, comme nous avons les mêmes principes je compte que nous tirerons les mêmes conséquences ....». Hocquart à Maurepas, Québec, 26 septembre 1747, A.C, C11A, 88: 38-39.

10 La Galissonière et Hocquart à Maurepas, Québec, 26 septembre 1747, AC, C11A, 87-1: 256-257. 
incursions aux environs de Louisbourg, et dans tous les lieux où il pourroit se rassembler des Anglois . . . ». ${ }^{11}$

Le gouvernement de la colonie est satisfait de la conduite des Indiens de la région du Lac des Deux-Montagnes. Il reconnaît le mérite de M. François Picquet qui, dans la direction de cette mission, obtient «l'estime et l'approbation de tout le monde ». A la suite de l'évêque de Québec Henri-Marie Du Breil de Pontbriand et du gouverneur Beauharnais, La Galissonière assure le ministre que le missionnaire Picquet ferait bon usage d'une «pension sur un bénéfice en France ». Il signale que ce secours sera d'autant plus nécessaire qu'il est possible que l'on ne puisse prélever sur le revenu des postes la gratification de 2,000 livres qui était destinée à cette mission. ${ }^{12}$

Dès le début de son administration, La Galissonière manifeste l'intention de détruire l'enclave britannique de Chouagen [Oswego], située au sud du lac Ontario. ${ }^{13}$ Il cherchera à profiter des circonstances pour mener à bien une entreprise qui dépend, notamment, des dispositions des Iroquois. Il souligne l'importance de la redoute de Chouagen avec une telle vigueur qu'il recourt à une expression exagérée, ce qui n'est pas fréquent dans sa correspondance: "Si ce fort reste aux anglois'a la paix, c'est une éternelle semence de discorde et un moyen sûr qu'auront toujours nos ennemis de débaucher nos sauvages. ${ }^{14}$ Plus tard, Pierre de Rigaud de Vaudreuil attribuera à Chouagen «la cause directe de tous les troubles survenus dans la colonie $\gg .^{15}$

11 Ibid., 258.

12 La Galissonière à Maurepas, Québec, 21 octobre 1747, AC, C11A, 87-2: 196-197.

13 La carte intitulée: «Entrée de la Rivière Chouégen 》 porte la note suivante: "Ce Plan a eté levé sur les lieux en 1749 par ordre de $\mathrm{M}^{\mathrm{r}}$ le Marquis de la Galissonière commandant Gnal en canada par le $\mathrm{S}^{\mathrm{r}}$ de Lery fils officier d'Infanterie et faisant les fonctions d'Ingenieur au dit Pays et remis au depôt par le même officier en 1752. 》 APC, Division des cartes, D 950 - Oswego - 1749; H. R. Holmden, Catalogue des cartes, plans et cartes marines conservés au dépôt des cartes des Archives Canadiennes (Ottawa, 1912), 3, no 22. 87-2: 198 .

14 La Galissonière à Maurepas, Québec, 21 octobre 1747, AC, C11A,

15 Lettre de Vaudreuil à Jean-Baptiste de Machault d'Arnouville, 24 juillet 1755. Citée par Guy Frégault, Le Grand Marquis, Pierre de Rigaud de Vaudreuil et la Louisiane (Montréal, 1952), 361. 
Outre les traitants de New York, de la Virginie et de la Caroline, ceux de la Pennsylvanie avaient dressé, à partir de 1744, des comptoirs au sud des Grands Lacs. ${ }^{16}$ Les présents des trafiquants britanniques aux indigènes, les menées des cinq Nations Iroquoises et «par dessus tout le manque de marchandises dans les Postes » ont provoqué du désordre. La Galissonière croit que les convois qui ont atteint Michillimakinac et Détroit rétabliront partiellement la paix. Selon lui, un des meilleurs moyens de prévenir les mouvements des Indiens serait de former une forte colonie agricole à Détroit, «seul moyen d'empêcher les Anglois de s'établir sur la belle Rivière [Ohio], établissement capable d'interrompre notre communication avec le Misissipi qui est presque également nécessaire aux deux colonies ». Détroit deviendrait le pivot de défense des postes avoisinants. La Galissonière proposait au ministre d'en stimuler le développement en accordant, pendant quelques années, aux habitants qui s'établiraient dans cette région le revenu de ce poste. ${ }^{17}$ La proposition de La Galissonière attirera l'attention du gouvernement métropolitain. $\mathrm{Au}$ printemps de 1749, quelques familles canadiennes s'établissent à Détroit; La Galissonière ne parvint à faire passer dans cette région qu'environ quarante-cinq personnes. ${ }^{18}$

A titre de gouverneur général, La Galissonière manifeste une vive sollicitude à l'égard de la défense de la colonie. Il réclame des renforts. Les soldats se marient en grand nombre; ils pourraient être avantageusement remplacés par des « recrues abondantes », ce qui renforcerait les troupes et faciliterait la colonisation militaire: "la colonie augmentera en hommes qui, quoiqu'ils ne soient plus dans les compagnies n'en seront pas moins de bons soldats dans le besoin. » Aux frontières, les postes manquent de garnison. Depuis le début de la guerre de la Succession d'Autriche, on y supplée par les habitants et les Indiens. Ce système de défense coûte cher; il est inefficace. On ne peut pas enrayer les abus. Les sauvages abandonnent leur chasse

16 G. Frégault, Ibid., 340.

$17 \mathrm{La}$ Galissonière à Maurepas, Québec, 22 octobre 1747, AC, C11A, 87-2: 199-200.

18 La Galissonière à Rouillé, Québec, 26 juin 1749, AC, C11A, 93: 141. 
et les habitants négligent l'agriculture. La Galissonière note que cette situation précaire produira ses effets longtemps après la fin des hostilités. Si les postes étaient suffisamment pourvus de troupes régulières, il serait possible, pense le gouverneur, de prendre l'offensive qui permettrait de récupérer en partie ce que l'on a perdu au Traité d'Utrecht. ${ }^{19}$

Émile Salone ${ }^{20}$ et Sylvain Girerd ${ }^{21}$ rapportent les données numériques contenues dans les dépêches de La Galissonière, en date du 22 octobre 1748 et du 20 septembre 1749: l'effectif militaire est faible. Le gouverneur réitère ses représentations auprès de la métropole afin d'obtenir une «nombreuse recrue » pour l'année $1748 .{ }^{22}$ Il commande des pièces d'artillerie, y compris quatre mille fusils pour compléter l'armement des milices. Les habitants doivent acheter leurs armes; plusieurs d'entre eux sont «très pauvres ». ${ }^{23}$ Le gouverneur ne se gênera pas de rappeler au ministre que «la guerre ne se fait nulle part sans dépenses ». Cette lapalissade lui valut une mise au point de la Cour: "On n'ignore point que la guerre ne se fait point sans qu'il en coûte; et c'est principalement pour cette raison que le Roy ne peut pas la faire partout. Sa $\mathbf{M}^{\text {té }}$ desire donc que vous vous en teniés aux dispo ons et aux mouvemens que vous jugerez necessaires pour mettre le Canada a couvert des entreprises que les Ennemis pourroient y tenter .... ${ }^{24}$ Ainsi, La Galissonière devra se limiter à un minimum de dépenses militaires.

Sur les instances des habitants de la colonie, le gouverneur de Beauharnais avait décidé, à la fin de 1745, d'ériger la fortification de Québec, aux frais du roi. Au début de l'année suivante, Maurepas lui faisait connaître que, de l'avis du roi, cette fortification pourrait avoir plus d'inconvénients que d'avantages.

19 Id. à Maurepas, Québec, 11 octobre 1747, AC, C11A, 87-2: 167-170. 20 La Colonisation de la Nouvelle-France (Paris, [1905]), 346. 1902), 12 .

21 L'Oeuvre militaire de La Galissonière au Canada (Saint-Etienne,

${ }_{22}$ La Galissonière à Maurepas, Québec, 3 novembre 1747, AC, C11A, 82-2: 214

23 Id. à Id., Québec, 21 octobre 1747, AC, C11A, 87-2: 193.

24 Cité par Guy Frégault, « Essai sur les finances canadiennes (17001750 », Revue d'Histoire de l'A mérique française, 13 (1959), 158. A l'avenir: $R H A F$. 
Louis XV remettait à «l'assemblée des officiers et des habitants principaux » le soin de juger s'il fallait démolir les ouvrages mis en chantier ou terminer la construction. L'évêque, les officiers militaires, le Séminaire, les Jésuites prennent parti pour l'édification d'une enceinte; les fonctionnaires civils et les marchands sont dissidents. Une remarque de Maurepas nous instruit du résultat de l'assemblée des notables: «ces $\mathrm{M}^{\text {rs }}$ n'ayant pas pris de parti décisif à cet égard et s'étant contentés de demander de nouveaux ordres sans donner de nouveaux éclaircissements, S. M. s'est trouvée dans la nécessité de s'en remettre encore à Eux. ${ }^{25}$ La Galissonière, autorisé par le roi à résoudre ce problème, ne tergiversera pas. C'est avec fermeté qu'il écrira: «Je crois sans hésiter que le party que l'on a pris de fortifier Québec étoit le meilleur, mais quand il y auroit eu alors du doute, il ne peut y en avoir aujourd'huy sur la nécessité de continuer ce travail avec activité... » Le gouverneur avise le ministre qu'il rendra compte conjointement avec Gilles Hocquart de la manière de financer cet ouvrage d'art.

Comme suite aux propositions des dirigeants de la colonie, la Cour avait déjà ordonné, le 23 janvier 1747, une hausse des droits d'entrée sur les vins et les alcools, pour une période de trois ans. L'édit de février 1748, qui frappait d'un impôt de $3 \%$ les articles d'importation au nombre de 305 et 59 articles d'exportation, est différé jusqu'à la fin des hostilités par une décision du Conseil d'État. Le Domaine perçoit, en plus des droits d'entrée, les revenus des congés et le produit de la ferme de divers postes de traite. ${ }^{26}$

Dès le début de son administration, La Galissonière s'occupe de l'activité économique de la colonie. Il recommande aux officiers, commandants dans les postes de «protéger les fermiers dans leurs traittes $\gg .^{27}$ Il trouve que la communication que l'intendant lui a donnée des dépêches du ministre sur les finances était «en quelque façon indispensable» puisque la majeure $351-352$

25 Maurepas à La Galissonière, Pontchartrain, 13 juin 1747, AC, B 86:

26 G. Frégault, $R H A F$., 13 (1959), 164.

27 La Galissonière et Hocquart à Maurepas, Québec, 7 octobre 1747, AC, C11A, 87-1: 278-279. 
partie des dépenses extraordinaires ressortit au gouverneur. ${ }^{28}$ La Galissonière réclame le fonds de 10,000 livres que le roi faisait distribuer aux familles pauvres. Après l'évêque de Québec et Philippe de Rigaud de Vaudreuil et Gilles Hocquart, il cherche à soulager la misère humaine: "vous adouciriez un détail qui par luy même est toujours triste », écrit-il au ministre. ${ }^{29}$

Le gouverneur insiste sur la nécessité d'obtenir les services de bons interprètes qui devraient être rémunérés convenablement. Les interprètes qui ne cherchent que leur profit personnel stimulent les Indiens à formuler des demandes exigeantes, ce qui constitue, selon La Galissonière, « une des principales causes de la dépense excessive» de cette colonie. Le gouverneur note qu'il serait «le plus souvent utile de se servir d'officiers pour interprètes, ils méritent la pluspart beaucoup plus de confiance, que des négocians qui ont avec les sauvages des liaisons d'intérêt...». Afin d'améliorer la politique indigène, il lui paraît «très important» d'encourager les officiers à apprendre les langues amérindiennes. ${ }^{30}$

Vers la fin de l'automne, La Galissonière accueille «avec grand plaisir » le compte rendu de la campagne de l'officier de Léry en Nouvelle-Angleterre: «je suis persuadé que la reussite en auroit été plus brillante si les sauvages avoient eu aussi bonne volonté que vous, mais il faut bien se contenter de tirer ce que l'on peut de ces gens là et je n'en suis pas moins content de votre zèle. ${ }^{31}$

Dans les dépêches qu'il adresse à Maurepas, à l'automne de 1747, La Galissonière avoue qu'il lui est impossible de rendre compte de la colonie aussi exactement qu'il le désirerait. Avant d'exercer les fonctions de gouverneur général, il était «très peu instruit » de l'état de la Nouvelle-France. Il est vrai que, depuis son arrivée, il prend connaissance de «plusieurs détails » d'admi-

28 La Galissonière à Maurepas, Québec, 24 octobre 1747, AC, C11A, 87-2: 204.

29 Id. à Id., Québec, 20 octobre 1747, AC, C11A, 87-2: 186-187.

30 Id. à Id., Québec, 23 octobre 1747, AC, C11A, 87-2: 201-203. de Québec.

31 Id. à de Léry, Québec, 25 novembre 1747. Archives de la province 
nistration..$^{32}$ Il avait précédemment informé le ministre d'avoir recours à l'expérience de son prédécesseur, le gouverneur de Beauharnais, au sujet des «bornes les plus convenables à établir pour le commerce du Canada et pour celui de la Louisiane, ainsi que de toutes les autres affaires de la colonie... ». Il profitait de l'occasion pour faire l'éloge de Honoré Michel de la Rouvillière, « extrêmement au fait de toutes les affaires de ce pays-cy, et à qui peu de choses auront échappé, étant laborieux, intelligent et fort attaché au service $\$^{33}$ Il continuera d'éprouver de vifs sentiments d'estime à l'égard de ce parent par alliance: «Vous m'avés appris le rétablissement de $\mathrm{M}$. Michel en mesme temps que sa maladie. On ne peut être plus reconnaissant que je suis des attentions que vous avés eu pour luy en cette occasion et en tout et je vous en demande la continuation. ${ }^{34}$

Le 24 octobre 1747, La Galissonière élabore les grandes lignes d'un programme qui préfigure le mémoire de décembre $1750 .^{35}$ Il reconnaît le fait de l'administration déficitaire de la Nouvelle-France; ce n'est pas une raison de négliger la colonie, «il y a en France beaucoup de places frontières qui ne produisent rien du tout, qui ont été bâties à grands frais et qui sont entretenues et gardées avec grande dépense ». La Galissonière prévoit une augmentation du commerce du blé, des chanvres et des pêcheries. Il insiste sur la richesse démographique qui passerait, avec les avantages matériels, aux Britanniques si l'on ne consolidait pas les frontières. Il cherche à diminuer les dépenses en assouplissant le mode de transfert de fonds. Dans le fonctionnement du système monétaire colonial, l'État métropolitain paie en monnaie de cartes les frais des produits, des denrées destinées aux troupes, du transport, des services. A l'automne, les négo-

32 Id. à Maurepas, Québec, 21 octobre 1747, AC, C11A, 87-2: 191.

33 Id. à Id., Québec, 11 octobre 1747, AC, C11A, 87-2: 172-173. Sur la carrière de Honoré Michel de la Rouvillière, voir Guy Frégault, Le Grand Marquis, 271-310.

34 La Galissonière à Vaudreuil, Paris, 23 janvier 1751, Huntington Library and Art Gallery, Loudoun Papers, $269 \mathrm{~A}$.

35 La Galissonière et Silhouette, "Mémoire sur les colonies de la France dans l'Amérique septentrionale », AC, C11A, 96: 175-212. Cf. Mémoires et Documents, Amérique, Ministère des Affaires Etrangères, 24-2 : 180-228. 
ciants portent au trésor royal ces cartes munies des signatures de l'intendant et du contrôleur de la Marine pour les dénominations supérieures. Ils reçoivent en retour des lettres de change que l'on présente au ministre de la Marine pour obtenir la monnaie métallique qui ne circule pas au Canada et qui sert au commerce en France. La monnaie de cartes n'a cours que dans la colonie.

La Galissonière est mis au courant de la situation financière par Gilles Hocquart qui est aux prises avec des difficultés provoquées par l'augmentation des dépenses, sans accroissement équivalent des revenus, qu'amplifie une économie de guerre. L'écart continue de s'élargir entre la somme des capitaux métropolitains expédiés au Canada et celle que nécessiteraient le développement de la colonie et les opérations militaires. ${ }^{36}$ Le gouverneur propose la solution suivante:

je n'en sais qu'un seul moyen qui est de donner aux cartes et au papier tout le crédit qu'ils peuvent avoir, ce qui ne se peut faire qu'en tirant sans aucune remise, ni difficulté, ni formalité, des lettres de change en un seul et court terme pour tout ce qui s'apportera au trésor; si cela étoit annoncé de bonne heure et que le trésorier tirât pour tout venant sans attendre le mois d'octobre, je suis sûr qu'on s'apercevroit dès la même automne d'une diminution considérable dans le prix des marchandises de France et des denrées du pays, et par conséquent dans les dépenses du Roy, diminution qui augmenteroit encore quand on sauroit que les lettres de change auroient été régulièrement acquittées malgré cette facilité. ${ }^{37}$

En face de l'expansion britannique, la France a besoin de sa colonie nord-américaine que l'on devrait « aider puissamment 》 au moyen de capitaux métropolitains: «je n'ignore pas, conclut La Galissonière, la difficulté des fonds en France et surtout pour la Marine, dans les circonstances présentes, mais cela n'empêche pas que je ne persiste dans mon opinion. ${ }^{38}$

36 G. Frégault, $R H A F ., 13$ (1959), 166-170.

37 La Galissonière à Maurepas, Québec, 24 octobre 1747, AC, C11A, 87-2 : 208-209. 38 Ibid., 210. 
Dans cette phase initiale de son gouvernement, à l'automne de 1747, Roland-Michel Barrin de La Galissonière prend contact avec la colonie et, le 3 novembre 1747, il informe le ministre Maurepas que ce sera au cours de l'année suivante qu'il pourra lui communiquer des renseignements précis sur la situation de la Nouvelle-France. ${ }^{39}$

Roland Lamontagne, Ph.D., Faculté des Arts,

Université de Montréal.

39 La Galissonière à Maurepas, Québec, 3 novembre 1747, AC, C11A, $87-2: 218$. 